Chem. Pharm. Bull.

35(12)4898-4906(1987)

\title{
Disposition Characteristics of Lipophilic Mitomycin C Prodrug in an Intra-arterial Muscle Infusion System
}

\author{
Toshiyuki Kakutani, Yumi Suematsu, Wing Yin Cheah, \\ ETSUKo Sumimoto, and Mitsuru Hashida*
}

\author{
Faculty of Pharmaceutical Sciences, Kyoto University, Yoshida \\ Shimoadachi-cho, Sakyo-ku, Kyoto 606, Japan
}

(Received May 15, 1987)

\begin{abstract}
A new experimental system was applied to obtain information about the local disposition of lipophilic mitomycin C (MMC) prodrugs after intra-arterial injection. Rabbit hind leg was perfused in situ using a single-pass technique and the outflow concentration-time curves of drugs following pulse injection were evaluated by using statistical moment analysis. Moment parameters (moments) were transformed to disposition parameters which express local disposition characteristics together with respect to elimination, distribution, and dispersion. Six lipophilic derivatives of MMC were tested together with the parent drug. Dimethylsulfoxide (DMSO) was used as a model injection vehicle that can be mixed with blood. Using this system, the following results were obtained. 1) Local disposition of the drug is decided by the balance of its affinities for intravascular and extravascular components. 2) Disposition can be modified by derivation of the drug to a more lipophilic prodrug. 3) Higher lipophilicity of a drug results in a larger organ clearance in the absence of albumin, but a smaller clearance in albumin-containing perfusate. 4) In the case of DMSO injection, intermediate clearance values are obtained regardless of the lipophilicity ef drugs, and it was suggested that DMSO decreases both partitioning from the injection vehicle to the tissue and plasma protein binding.
\end{abstract}

Keywords__ single-pass perfusion system; drug disposition; rabbit muscle tissue; mitomycin C; lipophilic prodrug; statistical moment analysis; intra-arterial injection; plasma protein binding; dimethylsulfoxide

Intra-arterial injection and local perfusion methods have been used in cancer chemotherapy since the $1950 \mathrm{~s}^{1)}$ An improvement in drug effectiveness and ultimate therapeutic success have been reported for these medical techniques. ${ }^{2)}$ Intra-arterial infusion is expected to offer a distinct advantage in comparison with common systemic administration; that is, it increases drug delivery to the area supplied by the cannulated artery and reduces systemic drug exposure. Although the theoretical justification for the advantage of intra-arterial infusion has been discussed, ${ }^{3)}$ exact information useful for pharmaceutical dosage design has not been obtained.

In the systemic administration mode, many attempts have been made to deliver cytotoxic drugs to the tumor site by means of drug delivery systems, ${ }^{4)}$ i.e., the utilization of physical devices, the chemical transformation of drug to prodrugs, ${ }^{5,6)}$ and combinations of the two. ${ }^{7}$ Recently, combined systems of physical devices and intra-arterial infusion have been developed as a chemo-embolization method, in which microcapsules,${ }^{8)}$ starch, ${ }^{9)}$ and oily Xray contrast medium (Lipiodol) ${ }^{10}$ ) are used as controlled release devices. However, application of the prodrug approach to local infusion has not been tried, although the lowmolecular-weight prodrug approach seems to be free from some of the disadvantages of colloid-size carriers.

In order to achieve precise chemotherapy through prodrug-based intra-arterial infusion, the effects of the physicochemical properties of drugs and the mode of injection should 
be clarified. We have established an experimental system in which rabbit hind leg is perfused in situ using a single-pass technique and data are analyzed based on statistical moment theory. ${ }^{11)}$ In previous papers, the effect of plasma protein binding on local drug disposition ${ }^{12)}$ and the regeneration characteristics of a prodrug ${ }^{13)}$ were investigated. In the present study, this system was used to elucidate the local disposition characteristics of lipophilic derivatives of mitomycin $\mathrm{C}(\mathrm{MMC})$ and the effects of the physicochemical properties of the drug and the injection vehicle were clarified.

\section{Experimental}

Materials-MMC was supplied by Kyowa Hakko Kogyo, Japan. Lipophilic MMC derivatives, propyloxycarbonyl MMC (propyl-MMC), benzyl MMC, benzyloxycarbonyl MMC (z-MMC), pentyloxycarbonyl MMC (pentyl-MMC), nonyloxycarbonyl MMC (nonyl-MMC), and cholesteryloxycarbonyl MMC (chol-MMC) were prepared as reported previously ${ }^{6}{ }^{6}$ The structures, physicochemical properties, and protein binding characteristics of these compounds are summarized in Table I. All other chemicals were of reagent grade and were obtained commercially (Nakarai Chemicals, Japan).

In Situ Perfusion of Rabbit Hind Leg - Figure 1 illustrates the whole animal experimental system. Details of the procedures were reported previously. ${ }^{11)}$ Male domestic rabbits weighing $1.99 \pm 0.13$ (mean \pm S.D.) kg were anesthetized with urethane, and the right legs were essentially isolated from the body by arterio-venous perfusion. The right iliolumbar vessels, common iliac vessels, and saphenous vein of the right hip were each ligated. The right femoral artery and vein were cannulated with vinyl tubing (i.d. $0.8 \mathrm{~mm}$, o.d. $1.2 \mathrm{~mm}$, Dural Plastics, Australia) and the inflow (arterial side) and outflow (venous side) cannula were kept at the level of the thigh during the experiment. In this preparation, there was almost complete recovery of the perfusate in venous outflow $(95.6 \pm 5.0 \%)$, and mixing of blood from the body was suppressed to $4.4 \pm 2.6 \%$. The weight of the perfused muscle was $50.9 \pm 8.0 \mathrm{~g}$.

Tyrode's solution (137 mM NaCl, $2.68 \mathrm{~mm} \mathrm{KCl}, 1.80 \mathrm{~mm} \mathrm{CaCl}, 11.9 \mathrm{~mm} \mathrm{NaHCO}, 0.362 \mathrm{mM} \mathrm{NaH}_{2} \mathrm{PO}_{4}$, $0.492 \mathrm{mM} \mathrm{MgCl}_{2}$, and $5.55 \mathrm{mM}$ D-glucose) with (system I) or without (system II) bovine serum albumin (Fraction V, Armour Pharmaceutical Co., U.K.) at a concentration of $4.7 \%(\mathrm{w} / \mathrm{v})$ was used as the perfusate. The perfusate $\left(37^{\circ} \mathrm{C}\right.$, gassed with $95 \% \mathrm{O}_{2}-5 \% \mathrm{CO}_{2}, \mathrm{pH} 7.4$ ) was pumped into the cannulated artery with the aid of a peristaltic roller pump at a rate of $1.66 \mathrm{ml} / \mathrm{min}$. Test compounds were dissolved in the perfusate or dimethylsulfoxide (DMSO) and injected into the line of perfusion flow via a six-position rotary valve injector (type 50 Teflon rotary valves, Rheodyne, CA). Test samples $(0.1 \mathrm{ml})$ were put into the flow as a pulse function by this procedure. When DMSO was used as an

TABle I. Structures, Physicochemical Properties and Protein Binding Characteristics of MMC and Lipophilic MMC Derivatives

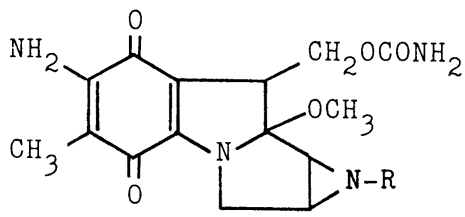

\begin{tabular}{|c|c|c|c|c|c|c|c|}
\hline \multirow[b]{2}{*}{ Compound } & \multirow[b]{2}{*}{$\mathbf{R}$} & \multirow[b]{2}{*}{$\mathrm{mp}\left({ }^{\circ} \mathrm{C}\right)$} & \multirow[b]{2}{*}{$P C_{\mathrm{oct}}^{a)}$} & \multicolumn{3}{|c|}{ Solubility $\left.(\mathrm{mM})^{b}\right)$} & \multirow{2}{*}{$\begin{array}{c}\text { Free fraction }^{c)} \\
\text { in system I } \\
(+ \text { albumin })\end{array}$} \\
\hline & & & & Water & $\begin{array}{c}\text { Sesame } \\
\text { oil }\end{array}$ & $\begin{array}{l}\text { Hexane } \\
\left(\times 10^{3}\right)\end{array}$ & \\
\hline MMC & $\mathrm{H}$ & $>270$ & 0.414 & 2.73 & 0.0180 & 0.234 & $0.907 \pm 0.0113$ \\
\hline Propyl-MMC & $\mathrm{COOC}_{3} \mathrm{H}_{7}$ & $203-207$ & 32.7 & 0.333 & 0.104 & $<0.02$ & $0.691 \pm 0.0170$ \\
\hline Benzyl MMC & $\mathrm{CH}_{2} \mathrm{C}_{6} \mathrm{H}_{5}$ & $119-121$ & 38.6 & 1.49 & 4.17 & 0.726 & $0.693 \pm 0.00732$ \\
\hline Z-MMC & $\mathrm{COOCH}_{2} \mathrm{C}_{6} \mathrm{H}_{5}$ & $102-104$ & 113 & 0.523 & 3.29 & 0.415 & $0.214 \pm 0.0162$ \\
\hline Pentyl-MMC & $\mathrm{COOC}_{5} \mathrm{H}_{11}$ & $89-93$ & 279 & 0.590 & 3.48 & 2.02 & $0.160 \pm 0.00320$ \\
\hline Nonyl-MMC & $\mathrm{COOC}_{9} \mathrm{H}_{19}$ & $139-141$ & 3670 & 0.00025 & 15.2 & 0.304 & $0^{d)}$ \\
\hline Chol-MMC & $\mathrm{COOC}_{27} \mathrm{H}_{45}$ & $140-142$ & 7610 & 0.00025 & 12.1 & 6.61 & 0 \\
\hline
\end{tabular}

a) Partition coefficient between $n$-octanol and water. b) Determined at $25^{\circ} \mathrm{C}$. c) Each value is the mean \pm S.D. of five experiments; details of determination methods were reported in ref. 12. d) Compounds can not be detected in the free form. 


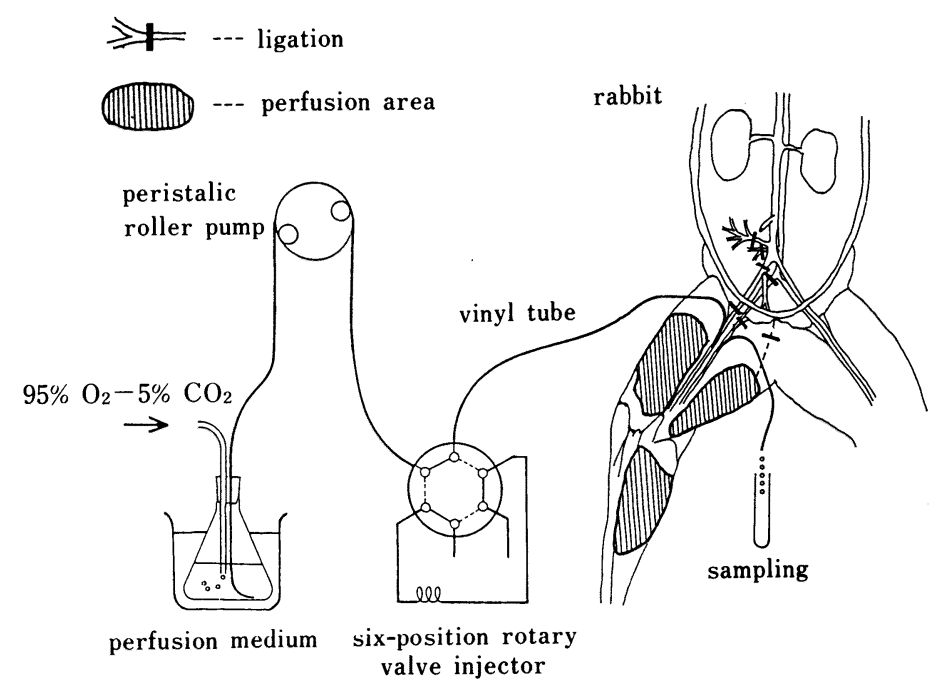

Fig. 1. In Situ Perfusion System of Rabbit Hind Leg

injection vehicle, Tyrode's solution containing albumin was used as the perfusate (system III).

Sampling and Assay - The details of the sampling and assay procedures were also described in the previous report. ${ }^{11)}$ The outflow perfusate was collected in weighed test tubes at appropriate time intervals (at first $1-2 \mathrm{~s}$, subsequently $10 \mathrm{~s}-2 \mathrm{~min}$ ). In the case of Evans blue and MMC, the concentration in the surpernate was measured after centrifugation for $15 \mathrm{~min}$ at $1500 \mathrm{rpm}$. The optical density of Evans blue was determined at $620 \mathrm{~nm}$. MMC was determined by measuring antimicrobial activity against Escherichia coli B using a disc-plate method. ${ }^{6,11)}$ Lipophilic derivatives were determined by high performance liquid chromatography (HPLC) in a reverse-phase mode after extraction with ethyl acetate. The stationary phase used was a Cosmosil $5 \mathrm{C}_{18}$ packed column $(4.6 \times 150 \mathrm{~mm}, \mathrm{Nakarai}$ Chemicals, Japan) and mixtures of methanol and distilled water were used as the mobile phase.

Moment Analysis - The present perfusion system is considered as a "black box" with linear disposition, and is denoted by the subscript $i$. The disposition function of this system is reflected in the output response to a unit pulse input, and is obtained by way of the concentration-time course in the outflow. The transit time of each drug molecule through the organ is statistically distributed according to the stochastic disposition process and the obtained outflow curve can be evaluated by moment analysis. The statistical concept of moments was introduced into pharmacokinetics by Yamaoka et al. ${ }^{14)}$ and Cutler, ${ }^{15)}$ and it has many applications in pharmacokinetic analysis as a modelindependent method. The first three (zero-th to second) statistical moment parameters (moments) are defined as follows;

$$
\begin{aligned}
& a u c_{i}=\int_{0}^{\infty} C_{i} d t \\
& \bar{t}_{i}=\int_{0}^{\infty} t \cdot C_{i} d t / a u c_{i} \\
& \sigma_{i}^{2}=\int_{0}^{\infty}\left(t-\bar{t}_{i}\right)^{2} \cdot C_{i} d t / a u c_{i}
\end{aligned}
$$

where $t$ is time and $C_{i}$ is the concentration of the substance normalized by expressing it as a fraction of the total injected amount in unit volume of outflow fluid (with dimensions of "\% of dose/ml"). $a u c_{i}, \bar{t}_{i}$, and $\sigma_{i}^{2}$ are the area under the outflow concenration-time curve, the mean transit time of the drug through the tissue, and the variance of the transit time, respectively.

The moments were calculated by numerical integration using the linear trapezoidal formula and extrapolation to infinite time based on a mono-exponential equation (infinite calculation method). ${ }^{11}$ ) The risk of error in moment calculation increases with the magnitude of the extrapolation required ${ }^{14,16)}$ and the approximate (finite) values of moments were also calculated for data points with concentrations higher than $0.3 \%$ of dose $/ \mathrm{ml}$ by using the linear trapezoidal rule (finite calculation method). ${ }^{11)}$ Vascular reference substance (VRS) is assumed neither to distribute to extravascular tissue nor to be eliminated, and a mixture of Evans blue and albumin was employed as the VRS. Calculation of Disposition Parameters from Moments- The calculation methods from moments, auc $c_{i}, \bar{t}_{i}$, and 
TABLE II. Derivation of the Disposition Parameters from Moments

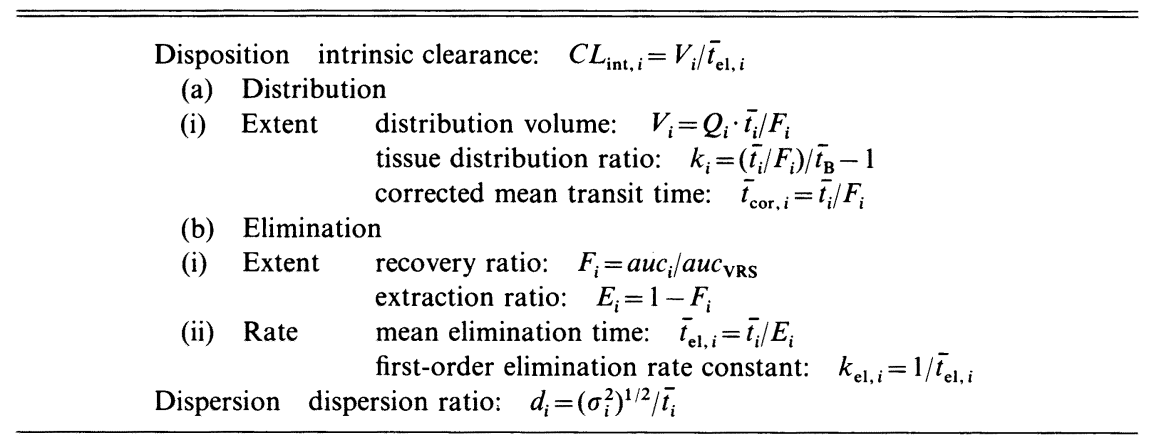

$Q_{i}$, flow rate of perfusion; $\bar{t}_{\mathrm{B}}$, mean transit time of VRS; $a u c_{\mathrm{VRS}}$, area under the curve of VRS.

\begin{abstract}
$\sigma_{i}^{2}$, to disposition parameters which express the local disposition characteristics of the drug are summarized in Table II, and the detailed theoretical background was reported in the previous paper. ${ }^{11)}$ The partition concept between mobile-stationary phases ${ }^{17)}$ and the plate theory ${ }^{18)}$ of chromatographic systems were introduced into the organ perfusion system. Derivation of disposition parameters from model-independent moments was carried out based on either of the following physiological models.

1) The well-stirred model ${ }^{19,20)}$ (one-compartment perfusion model ${ }^{21,22)}$ ).

2) A multiphase mamillary compartment model in which only the central compartment possesses the elimination process (as represented by the two-compartment perfusion model ${ }^{3,22)}$ ).

$C L_{\text {int }, i}$ is the intrinsic clearance. ${ }^{19,20)} k_{i}$ (tissue distribution ratio) is the ratio of mass of drug in tissue to that in perfusate under steady-state conditions and corresponds to the capacity factor in a chromatographic system. ${ }^{17)} \bar{t}_{\text {cor, } i}$ (corrected mean transit time) is the true value of mean transit time of drug molecules and corresponds to the retention time. ${ }^{17)} F_{i}$ (recovery ratio) and $E_{i}$ (extraction ratio) represent the ratios of outflowed and extracted drug amounts to input dose, respectively. $\bar{t}_{\mathrm{el}, i}$ (mean elimination time) is mean time value of drug elimination and corresponds to mean residence time (MRT) in a whole-body recirculation system. ${ }^{14,16)}$ The dispersion ratio, $d_{i}$, gives information for selecting the preferred model. When model 1 or 2 is valid, the dispersion ratio takes a value of 1 or more than 1 , respectively. In the case of insufficient mixing, the value of $d_{i}$ becomes less than 1 . In particular, when $d_{i}$ equals zero, it represents a condition of plug flow in which mixing in the system can be neglected (the parallel tube model ${ }^{20)}$ ).
\end{abstract}

\title{
Results
}

\section{Outflow Patterns and Moments}

Figure 2 illustrates typical concentration-time curves of pentyl-MMC. In Fig. 2A, the results in the initial $3 \mathrm{~min}$ are plotted on a normal scale; Fig. 2B shows the whole concentration-time curves over $30 \mathrm{~min}$ plotted on a semilogarithmic scale. The concentration of pentyl-MMC rose rapidly to a peak, then fell off in a manner described by a sum of exponentials. As compared with system I (+albumin), system II (-albumin) showed a diminution in outflow concentrations, but the slopes in the terminal phase were essentially similar. The outflow pattern of system III (DMSO) was rather closer to that of system II than to that of system $I$ in the first $3 \mathrm{~min}$, but showed the fastest decline in the terminal phase among them.

The outflow patterns of other compounds in the case of system II also showed diminutions in peak concentration relative to those of system I, and system III usually gave intermediate patterns between systems I and II. The differences between systems I and II were attributable to the free fractions in system $I$.

The outflow patterns of test compounds in each system were evaluated by moment analysis. Table III lists the moments of all compounds for system III with the moments of the VRS. Although a derivative with higher lipophilicity passed more slowly in system II and more quickly in system $\mathrm{I},{ }^{12}$ the lipophilicity had no special influence in the case of DMSO injection. Because the moments are complex parameters reflecting all the disposition character- 

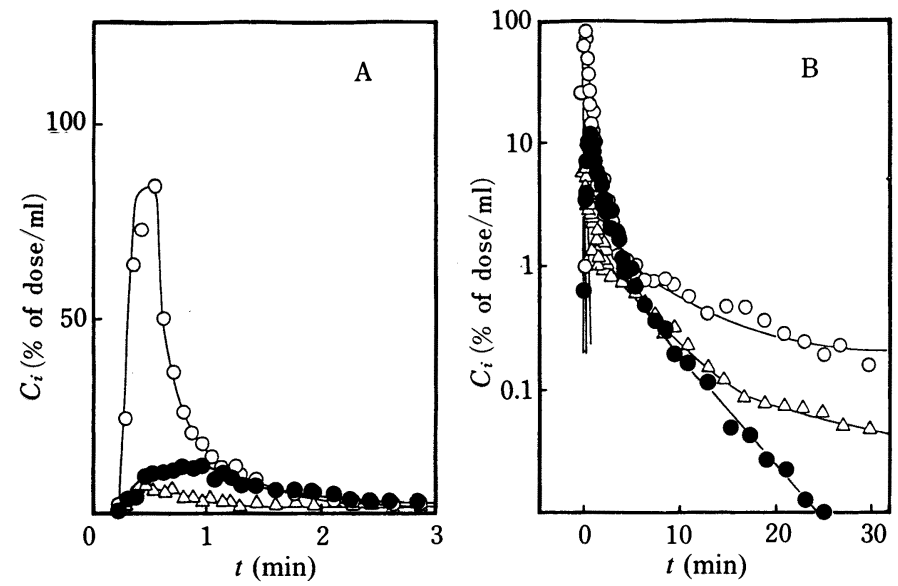

Fig. 2. Typical Outflow Concentration-Time Curve of Pentyl-MMC

O, system I (+albumin); $\triangle$, system II (-albumin); •, system III (DMSO). A, initial $3 \mathrm{~min}$; B, whole period.

TABLE III. Moment Parameters (Moments) for VRS and MMC Derivatives in System III (DMSO)

\begin{tabular}{lllcc}
\hline \hline Compound & Moments & $\begin{array}{c}a u c_{i} \\
(\% \text { of dose } \cdot \mathrm{min} / \mathrm{ml})\end{array}$ & $\begin{array}{c}\bar{t}_{i} \\
(\mathrm{~min})\end{array}$ & $\begin{array}{c}\sigma_{i}^{2} \\
\left(\mathrm{~min}^{2}\right)\end{array}$ \\
\hline VRS (6) & Infinite & $53.9 \pm 4.20$ & $1.11 \pm 0.114$ & $1.60 \pm 0.619$ \\
& Finite & $53.3 \pm 3.75$ & $1.02 \pm 0.157$ & $0.946 \pm 0.363$ \\
MMC (5) & Infinite & $26.9 \pm 5.83$ & $4.05 \pm 0.186$ & $40.2 \pm 9.28$ \\
Propyl-MMC (5) & Finite & $24.2 \pm 5.39$ & $2.32 \pm 0.193$ & $6.35 \pm 1.13$ \\
& Infinite & $16.5 \pm 1.67$ & $3.12 \pm 0.274$ & $50.6 \pm 61.6$ \\
Benzyl MMC (5) & Finite & $15.3 \pm 1.60$ & $1.91 \pm 0.210$ & $2.66 \pm 0.769$ \\
& Infinite & $19.0 \pm 1.66$ & $3.04 \pm 0.225$ & $19.2 \pm 3.63$ \\
Z-MMC (5) & Finite & $17.4 \pm 1.57$ & $1.98 \pm 0.247$ & $3.77 \pm 0.989$ \\
& Infinite & $19.8 \pm 3.30$ & $2.84 \pm 0.127$ & $22.7 \pm 8.64$ \\
Pentyl-MMC (5) & Finite & $17.6 \pm 3.10$ & $1.57 \pm 0.198$ & $1.79 \pm 0.466$ \\
& Infinite & $21.3 \pm 2.29$ & $3.20 \pm 0.152$ & $37.6 \pm 19.7$ \\
Nonyl-MMC (5) & Finite & $19.6 \pm 2.34$ & $1.98 \pm 0.180$ & $2.83 \pm 0.642$ \\
& Infinite & $16.6 \pm 1.96$ & $11.5 \pm 1.18$ & $388 \pm 177$ \\
Chol-MMC (5) & Finite & $12.0 \pm 1.78$ & $2.98 \pm 0.321$ & $5.66 \pm 1.00$ \\
& Infinite & $3.85 \pm 1.84$ & $5.75 \pm 1.14$ & $78.9 \pm 71.6$ \\
& Finite & $2.28 \pm 1.51$ & $1.14 \pm 0.407$ & $0.831 \pm 0.809$ \\
\hline
\end{tabular}

Each value is the mean \pm S.D., with the number of experiments in parenthesis.

istics, it seemed difficult to analyze the lipophilicity-disposition relationship only in terms of the first three moments.

\section{Relationships between Disposition Characteristics and Lipophilicity of Drugs}

The disposition parameters were derived independently from moments by using the equations in Table II, and they allowed the detailed evaluation of tissue disposition characteristics. Figures 3-5 show the relationships of calculated extraction ratio $\left(E_{i}\right)$, tissue distribution ratio $\left(k_{i}\right)$, and mean elimination time $\left(\bar{t}_{\mathrm{el}, i}\right)$ to the $P C_{\mathrm{oct}}$ values of the tested compounds. These values were obtained from the mean values of infinite moments and the results from finite moments were essentially similar to them.

Concerning $E_{i}$ values, the following conclusions may be drawn (Fig. 3). Higher 


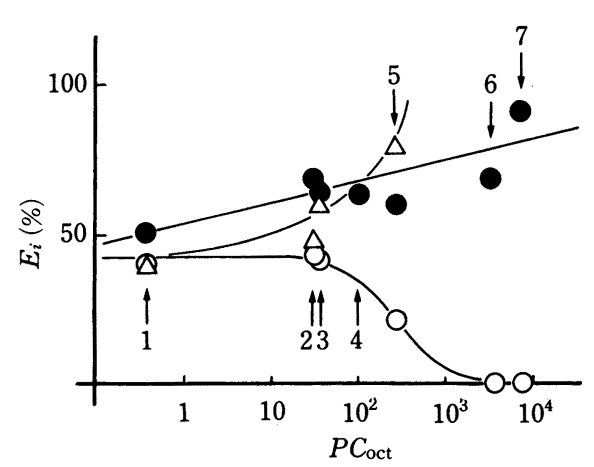

Fig. 3. Relationships between Extraction Ratio, $E_{i}$, and Partition Coefficient ( $n$-Octanol/ Water), $P C_{\text {oct }}$

O, system I (+albumin); $\triangle$, system II (-albumin); system III (DMSO).

1, MMC; 2, propyl-MMC; 3, benzyl MMC; 4, ZMMC; 5, pentyl-MMC; 6, nonyl-MMC; 7, cholMMC.

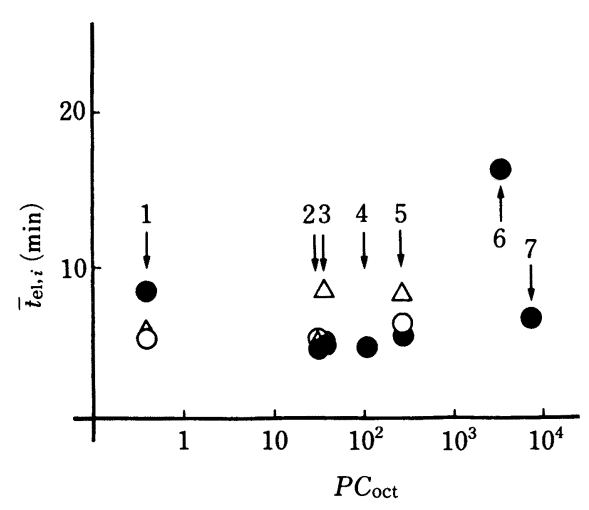

Fig. 5. Relationships between Mean Elimination Time, $\bar{t}_{\mathrm{el}, i}$, and Partition Coefficient ( $n$ Octanol/Water), $P C_{\text {oct }}$

O, system I (+albumin); $\triangle$, system II (-albumin); , system III (DMSO).

1, MMC; 2, propyl-MMC; 3, benzyl MMC; 4, ZMMC; 5, pentyl-MMC; 6, nonyl-MMC; 7 , cholMMC.

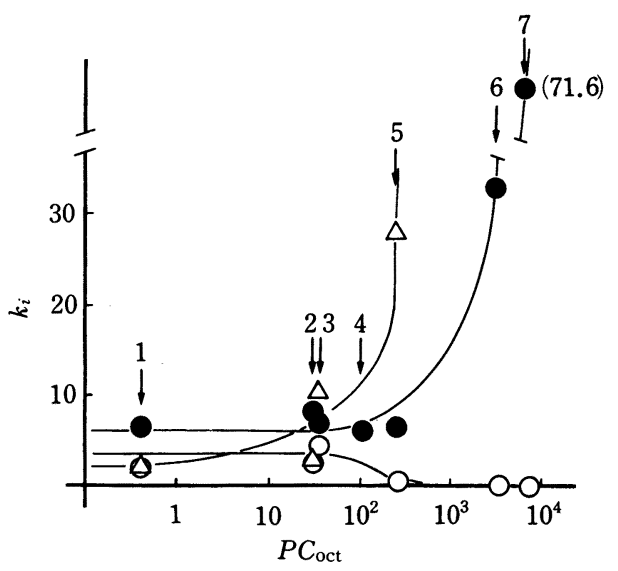

Fig. 4. Relationships between Tissue Distribution Ratio, $k_{i}$, and Partition Coefficient ( $n$ Octanol/Water), $P C_{\text {oct }}$

$O$, system I (+albumin); $\triangle$, system II (-albumin); , system III (DMSO).

1, MMC; 2, propyl-MMC; 3, benzyl MMC; 4, ZMMC; 5, pentyl-MMC; 6, nonyl-MMC; 7, cholMMC.

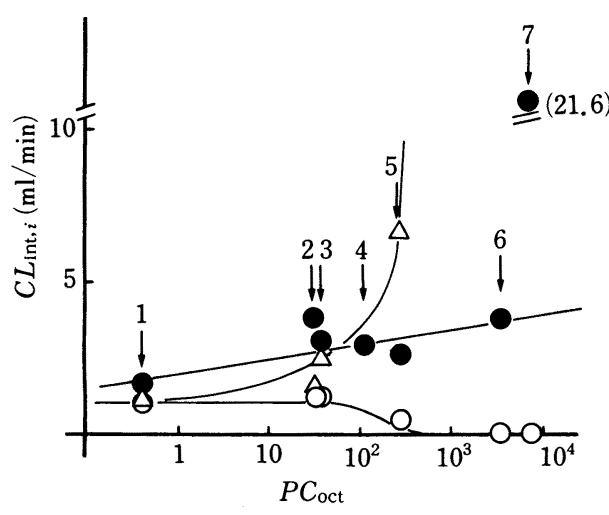

Fig. 6. Relationships between Intrinsic Clear-

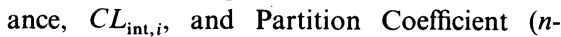
Octanol/Water), $P C_{\text {oct }}$

O, system I (+albumin); $\triangle$, system II (-albumin); 0 , system III (DMSO).

1, MMC; 2, propyl-MMC; 3, benzyl MMC; 4, ZMMC; 5, pentyl-MMC; 6 , nonyl-MMC; 7, cholMMC.

lipophilicity resulted in larger elimination in the absence of albumin in the intravascular space. Plasma protein binding decreased the organ extraction, and in the presence of albumin, higher lipophilicity resulted in lower elimination. It is possible that the decrease of the colloidal osmotic pressure enlarged the distribution volume in system II. However, the difference in organ extraction between the cases with and without albumin was mainly determined by the free fraction of drug in each system. In the case of DMSO injection, the organ extraction was not influenced so much by lipophilicity, and it was suggested that the effects of lipophilicity on the distribution to the extravascular space and on the binding to intravascular albumin 
canceled each other out. In addition, DMSO enhanced the organ uptake of relatively weakly lipophilic compounds such as MMC and propyl-MMC (against systems I and II; $p<0.05$ by Student's $t$-test).

The value of $k_{i}$ showed the same dependency on $P C_{\text {oct }}$ as $E_{i}$ in each system (Fig. 4). Highly lipophilic derivatives, nonyl-MMC and chol-MMC, showed high tissue distribution even in DMSO solution, suggesting that albumin binding was restricted by their waterinsolubility. Although the extent of elimination was dependent on lipophilicity, the elimination rates $\left(\bar{t}_{\mathrm{el}, i}\right)$ were constant in all cases (Fig. 5).

Figure 6 illustrates the relationships between $P C_{\text {oct }}$ and intrinsic clearance. Because the elimination rates were independent of lipophilicity (Fig. 5), this parameter seemed to be mainly determined by the distribution extent (intrinsic clearance is expressed as $\left.V_{i} / \bar{t}_{\mathrm{el}, i}\right)$. Dispersion ratios greater than 1 were obtained for all cases, indicating that the necessary condition for calculation in Table II was attained.

\section{Discussion}

In this paper, we have examined the local disposition characteristics of MMC prodrugs, focusing on their quantity. The muscle was used as the model tissue since it has a continuous capillary endothelium and occupies a large part of the body. DMSO was used as the model vehicle for elucidating the effect of vehicle properties; although DMSO is not practically used, it can dissolve even highly lipophilic drugs and mix with perfusate. The present results suggested that drug molecules should be transformed to more lipophilic forms with the smallest possible affinity for intravascular albumin, in order to achieve effective local perfusion therapy. The possibility for controlling tissue distribution by the selection of an injection vehicle with appropriate physicochemical properties was also demonstrated. Concerning the regeneration process of MMC from the prodrug, which is crucial for the therapeutic efficacy of the present modality, the results of pharmacokinetic analysis will be reported together with a new theoretical approach. ${ }^{13)}$

Miyauchi et al. expressed the "apparent" intrinsic clearance in terms of the intrinsic clearances for the influx, efflux, and sequestration processes as follows. ${ }^{23)}$

$$
C L_{\mathrm{int}, i}=\frac{C L_{\mathrm{int}, \mathrm{seq}} \times C L_{\mathrm{int}, \mathrm{inf}}}{C L_{\mathrm{int}, \mathrm{seq}}+C L_{\mathrm{int}, \mathrm{eff}}}
$$

If $C L_{\mathrm{int}, \mathrm{seq}} \ll C L_{\mathrm{int}, \text { eff }}$ is not attained, the contribution of $C L_{\mathrm{int}, \text { inf }}$ to $C L_{\mathrm{int}, i}$ can not be neglected. That is, the influx rate into the extravascular space is largely reflected in the elimination rate from the perfusate, in the case of a relatively slow efflux rate. For propylMMC, it was confirmed that the in vitro sequestration rate was smaller than the elimination rate from the perfusate compartment. ${ }^{13)}$ Whereas the lipophilic derivatives show a wide variation in in vitro stability, ${ }^{6}$ ) their mean elimination times from the perfusate were approximately constant (Fig. 5). These facts suggest that the derivatives are eluted slowly from the extravascular space of the muscle tissue. A large part of the extraction ratio corresponds to the ratio of drug which is diffused into the extravascular space in a short time and will be recovered in outflow fluids according to an irreversible (non-linear) process.

The most distinctive feature of this experimental system is the application of statistical moment analysis to a local perfusion single-pass system. In the field of physiology, numerous analytical models have been proposed for a local perfusion system. However, such models are sometimes more sophisticated than is warranted by the experimental data. ${ }^{11.24)}$ In this investigation, a simple model is used only for the derivation of the physiologically meaningful parameters from moments. Each process occurring in this system is evaluated with statistical concepts of sum, mean, and variance, and the obtained parameters are free from restrictions 
arising from complex modeling. Thus, the moment analysis enabled us to evaluate the disposition characteristics of drugs in a local perfusion system, and the effects of the physicochemical properties of drugs on their disposition were elucidated.

In moment analysis, the terminal slope extrapolation produces calculation errors. It is not easy to choose a starting point for the terminal phase or to discriminate between the terminal phase and the subsequent apparent plateau phase. The finite calculation method is free from such errors. The lipophilicity-disposition relationships derived from the finite moments were essentially similar to those from the infinite moments, though there was some underestimation of moments (Table III). ${ }^{11,16)}$ Consequently, the values of $V_{i}, k_{i}, \bar{t}_{\mathrm{cor}, i}, F_{i}, \bar{t}_{\mathrm{el}, i}$, and $d_{i}$, were underestimated and the values of $C L_{i \mathrm{nt}, i}, E_{i}$, and $k_{\mathrm{el}, i}$ were overestimated. The finite moment calculation is useful for relative evaluation. However, the infinite moments are necessary for quantitative evaluation, and a suitable criterion for accurate determination of the terminal slope is required.

Concerning the impulse response of a single-pass system observed as a log-convex disposition curve, Weiss has recently proved that it has the mono-exponential terminal phase; this supports the validity of the moment calculation. ${ }^{25)}$ Wide applicability of statistical moment analysis to single-pass dilution systems has already been suggested by Yamaoka and Nakagawa. ${ }^{26)}$ Because the moments are defined by transfer functions using Laplace transformation, network theory can also be applied to this analytical system. ${ }^{27}$ Based on network theory, we have elucidated the effect of plasma protein binding on tissue distribution based on the results for systems I and II, and the general assumption that only the unbound form is available for organ uptake was confirmed. ${ }^{12)}$ In addition, Homer and Weathersby had calculated the moment values for complicated models constructed with various assumptions such as well-stirred, plug flow, radial diffusion, and axial diffusion. ${ }^{28)}$ They indicated that the moment analysis of experimental crude data would give fundamental information for model selection. Based on these considerations, statistical moment analysis can be concluded to be a useful tool for analyzing drug disposition at the organ level and for devising systematic strategies for the development of a new dosage modality.

Acknowledgements The authors thank Professor Hitoshi Sezaki, Faculty of Pharmaceutical Sciences, Kyoto University, Kyoto, Japan for his valuable suggestions and constructive discussions during this work. A part of this work was supported by a Grant-in-Aid for Scientific Research from the Ministry of Education, Science, and Culture, Japan.

\section{References and Notes}

1) C. T. Klopp, T. C. Alford, J. Bateman, G. N. Berry, and T. Winship, Ann. Surg., 132, 811 (1950); O. Creech, Jr., E. T. Krementz, R. F. Ryan, and J. N. Winblad, ibid., 148, 616 (1958).

2) D. Jinnai, H. Mogami, H. Higashi, T. Hayakawa, N. Kanari, and R. Yamada, Brain Nerve, 19, 333 (1967); Y. Inuyama, "Mitomycin C: Current Status and New Developments," ed. by S. K. Carter and S. T. Crooke, Academic Press, New York, 1979, p. 173.

3) W. W. Eckman, C. S. Patlak, and J. D. Fenstermacher, J. Pharmacokinet. Biopharm., 2, 257 (1974).

4) R. L. Juliano, “Drug Delivery Systems," ed. by R. L. Juliano, Oxford University Press, New York, 1980, p. 3; G. Gregoriadis, Nature (London), 265, 407 (1977).

5) Y. Takakura, S. Matsumoto, M. Hashida, and H. Sezaki, Cancer Res., 44, 2505 (1984).

6) H. Sasaki, E. Mukai, M. Hashida, T. Kimura, and H. Sezaki, Int. J. Pharmaceut., 15, 49 (1983); H. Sasaki, M. Fukumoto, M. Hashida, T. Kimura, and H. Sezaki, Chem. Pharm. Bull., 31, 4083 (1983).

7) M. Hashida and H. Sezaki, "Microspheres and Drug Therapy. Pharmaceutical, Immunological and Medical Aspects," ed. by S. S. Davis, L. Illum, J. G. McVie, and E. Tomlinson, Elsevier Biomedical Press, New York, 1984, p. 281; H. Sasaki, T. Kakutani, M. Hashida, and H. Sezaki, J. Pharm. Pharmacol., 37, 461 (1985); H. Sasaki, T. Kakutani, M. Hashida, T. Kimura, and H. Sezaki, Chem. Pharm. Bull., 33, 2968 (1985).

8) R. Nemoto and T. Kato, "Microspheres and Drug Therapy. Pharmaceutical, Immunological and Medical Aspects," ed. by S. S. Davis, L. Illum, J. G. McVie, and E. Tomlinson, Elsevier Biomedical Press, New York, 
1984 , p. 229.

9) W. D. Ensminger, J. W. Gyves, P. Stetson, and S. W. Andrews, Cancer Res., 45, 4464 (1985).

10) K. Iwai,.H. Maeda, and T. Konno, Cancer Res., 44, 2115 (1984).

11) T. Kakutani, K. Yamaoka, M. Hashida, and H. Sezaki, J. Pharmacokinet. Biopharm., 13, 609 (1985).

12) T. Kakutani, E. Sumimoto, and M. Hashida, J. Pharmacokinet. Biopharm., in press.

13) T. Kakutani, R. Atsumi, E. Sumimoto, and M. Hashida, Chem. Pharm. Bull., 35, 4907 (1987).

14) K. Yamaoka, T. Nakagawa, and T. Uno, J. Pharmacokinet. Biopharm., 6, 547 (1978).

15) D. J. Cutler, J. Pharm. Pharmacol., 30, 476 (1978).

16) S. Riegelman and P. S. Collier, J. Pharmacokinet. Biopharm., 8, 509 (1980).

17) L. R. Snyder and J. J. Kirkland, "Introduction to Modern Liquid Chromatography," 2nd ed., John Wiley and Sons, Inc., New York, 1979, p. 15.

18) A. B. Littlewood, "Gas Chromatography. Principles, Techniques, and Application," 2nd ed., Academic Press, New York, 1970, p. 144.

19) M. Rowland, L. Z. Benet, and G. G. Graham, J. Pharmacokinet. Biopharm., 1, 123 (1973).

20) K. S. Pang and M. Rowland, J. Pharmacokinet. Biopharm., 5, 625 (1977).

21) M. Weiss and W. Förster, Eur. J. Clin. Pharmacol., 16, 287 (1979).

22) M. Weiss, J. Math. Biol., 15, 305 (1982).

23) S. Miyauchi, Y. Sugiyama, Y. Sawada, K. Morita, T. Iga, and M. Hanano, J. Pharmacokinet. Biopharm., 15, 25 (1987).

24) M. Silverman and C. Trainor, Fed. Proc., Fed. Am. Soc. Exp. Biol., 41, 3054 (1982); J. B. Bassingthwaighte, ibid., 41, 3040 (1982).

25) M. Weiss, J. Theor. Biol., 116, 355 (1985); M. Weiss, J. Pharmacokinet. Biopharm., 14, 635 (1986).

26) K. Yamaoka and T. Nakagawa, J. Chromatogr., 93, 1 (1974); idem, ibid., 117, 1 (1976).

27) R. L. Schoenfeld, Ann. N. Y. Acad. Sci., 108, 69 (1963); A. Rescigno and G. Segre, "Drug and Tracer Kinetics," Blaisdell, Massachusetts, 1966, p. 75.

28) L. D. Homer and P. K. Weathersby, J. Theor. Biol., 87, 349 (1980). 